\title{
The effect of cemented femoral stem cross- section geometry in total hip replacement
}

\author{
A. Ramos', F. Fonseca' \& J. A. Simões' \\ 'Departamento de Engenharia Mecânica, Universidade de Aveiro, \\ Portugal \\ ¿Serviço de Ortopedia A. Hospitais da Universidade de Coimbra. \\ Portugal
}

\begin{abstract}
This paper describes the finite element analysis on the effect of stem crosssectional geometries on stresses generated at the bone-cement and cement-stem interfaces. A simplified numerical model of the implanted femur was used, were the femur was modelled as a tubular structure and the femoral component modelled as an extruded beam. All materials were assumed isotropic and linearly elastic. The cross-sections were assumed symmetric about the medial-lateral axis. A layer of constant thickness of cement was interposed between the stem and the femoral cortex and a complex system of forces and moments generated by the hip joint contact force was applied. Maximum and minimum principal stresses were determined for twelve different cross-sectional geometries. The results show that organic type geometries provoke, comparatively, lower tensile and compression stresses. Rigid (sharp) geometries and cross-sections of low second moment of area provoke much higher interface stresses.
\end{abstract}

\section{Introduction}

Clinical experience with total hip replacement has been characterised by a variety of mechanical (dislocation, wear, component failure and loosening) [1] and biological problems. Surgeons and engineers have been involved in the design of total hip replacements for many years and new designs have been launched into market claiming the solution of problems inherent in pre-existing ones [1]. Within the cemented stem, the femoral component transmits the joint reaction force through the cement to the proximal femur. Several design factors can influence the stress-strain distribution and thus the longevity of the hip replacement: stem size, stem cross-section shape, stem frontal plane shape, stem 


\section{4}

Simulations in Biomedicine $V$

length, cement thickness and cement material properties and the nature of the stem-cement and cement-bone bonds [1].

The introduction of polymethylmethacrylate (PMMA) in orthopaedic surgery to anchor implants to the surrounding bone was one of the most relevant history facts in total hip replacement. PMMA was used with success by Sir John Charnley to fix both femoral and acetabular components. The cement mantle avoids high interface bone-implant micromovements and due to its elasticity, a more uniform stress transfer occurs. A negative aspect in the use of cemented prostheses is related to its collateral effects, referred in clinical reports as "cement disease", and therefore responsible for prostheses loosening. However, the anchoring of the implant to the bone with cement resolved partially the micro movements' problem at the interface. One of the most common modes of femoral component loosening is the loss of proximal support [2].

A cemented hip replacement originates two different interfaces: cement-stem and bone-cement interfaces. Cement is a material of poor resistance properties and its failure is generally referred to a fatigue and/or accumulated damage process. Many researchers have characterised the static and dynamic tensile and compressive strength of cement. Lee et al. [3] determined with laboratoryprepared specimens values of $25 \mathrm{MPa}$ and $77 \mathrm{MPa}$ for ultimate tensile and compressive strengths respectively. Cement fatigue failure is potentially a more serious problem. In fact, Freitag et al. [4] reported fatigue life of PMMA laboratory-prepared specimens to be about one million cycles. Fuch [5] presents in his study more realistic cement fatigue strength, since a triaxial stress state would cause fatigue failure of the cement in vivo at one million cycles and would be half of the fatigue strength of axially loaded laboratory specimens. Cement material in hip joint reconstructions plays and important key goal in the performance of the femur-cement-stem construction.

This paper describes the preliminary research done that aims the development of a novel cemented hip femoral component. The effect of cemented stem geometries on the stresses at the bone-cement and cement-stem interfaces was critically assessed using a simplified three-dimensional femur-cement-prosthesis construction. Other investigators have used this type of simplified model, since it allows a much easier understanding of the load transfer mechanism.

\section{Materials and methods}

A simplified three-dimensional numerical model of the cemented implanted femur was used. The femur was modelled as a tubular structure and the femoral component as a beam using Solid Works $® 2001$ software. Both were assumed of isotropic and linearly elastic material (elastic modulus of the femur and stem equal to $20 \mathrm{GPa}$ and $210 \mathrm{GPa}$ respectively; Poisson's ratio equal to 0.3 ). A cement mantle (elastic modulus equal to $3 \mathrm{GPa}$ and Poisson's ratio equal to 0.28) of $2 \mathrm{~mm}$ thickness was interposed between the femur and the stem. All cross- 
sections were assumed symmetric about the medial-lateral axis. A perfect bond between the implant and the cement mantle and the surrounding bone structure was assumed, therefore no friction was considered. Figure 1 shows the finite element mesh and the schematic drawing of the simplified bone-stem construction.
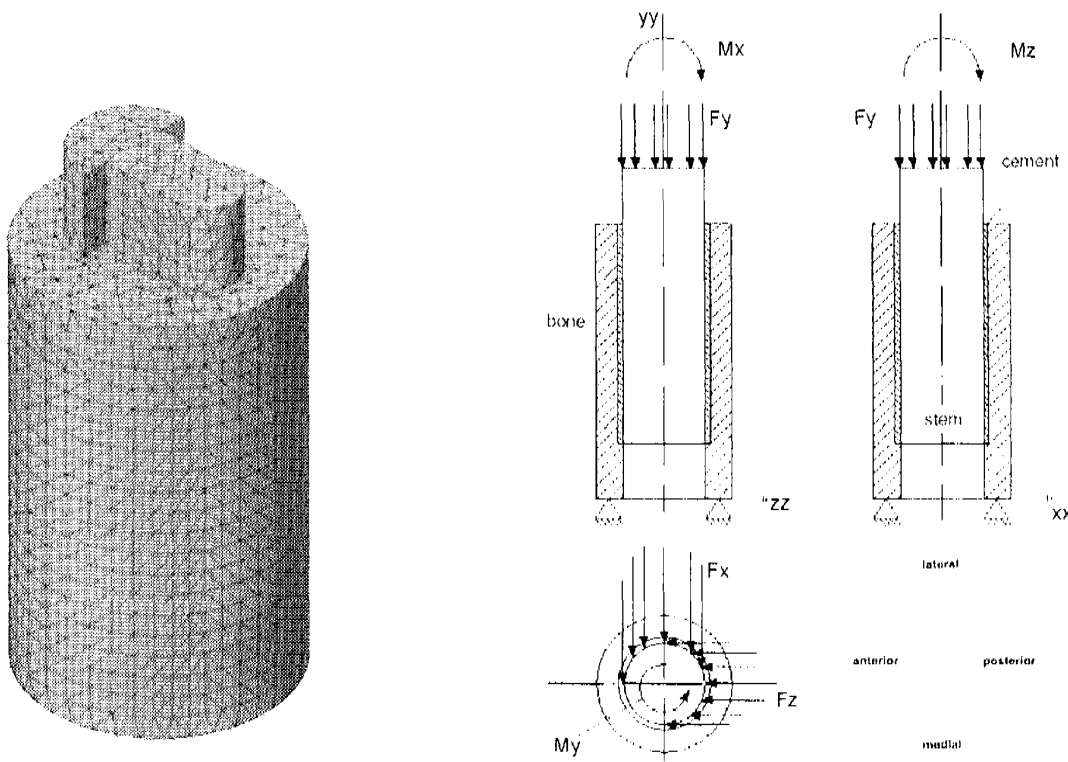

Figure 1: Finite element mesh and schematic drawing of the simplified cemented hip replacement.

Twelve different geometric sections (figure 2) were considered and studied in this work. The geometric sections were taken from proximal transverse sections of cemented commercial prostheses (e.g. Charnley, Freeman, Stanmore...) and from those presented in ref. [1]. Other geometries were idealised based on what we designated by "organic" (smooth) and "rigid" (sharp) geometries. A purely cylindrical stem of circular geometry (section \#12), of academic nature, was used as a reference one. The contact area of all geometries between the stem and the cement mantle were very similar. Table 1 shows the cross section area and second moment of area in the three principal directions for all geometries studied. The differences of areas relatively to section \#12 are identified in parenthesis.

The simulations were performed with Cosmos Works $\otimes 6.0$ finite element analysis software. The meshes of all femur-stem constructions were of identical number of nodes and elements (parabolic tetrahedral element), an average of 30000 nodes and 20000 elements. The boundary conditions were set-up considering the femur bone rigidly fixed at its distal region and the loads were 


\section{6}

Simulations in Biomedicine $V$

applied proximally accordingly to Huiskes [5]. The loading configuration was composed of a normal load ( $\mathrm{Fy}$ ), transverse loads ( $\mathrm{Fx}$ and $\mathrm{Fz}$ ), bending moments ( $\mathrm{Mx}$ and $\mathrm{Mz}$ ) and a torsional moment (My). Following Kuiper and Huiskes [6], which considered a hip joint reaction of $2460 \mathrm{~N}$, the forces applied within the study were: $\mathrm{Fx}=862 \mathrm{~N}, \mathrm{Fy}=2228 \mathrm{~N}$ and $\mathrm{Fz}=99 \mathrm{~N}$ (see figure 1). The bending and torsional moments were determined considering a $30 \mathrm{~mm}$ distance from the application of the forces relatively to the stem's axis. For the analysis of the results, the lateral, medial, anterior and posterior aspects of the sections of the stems must be considered similar to section \#2 shown in figure 1 .
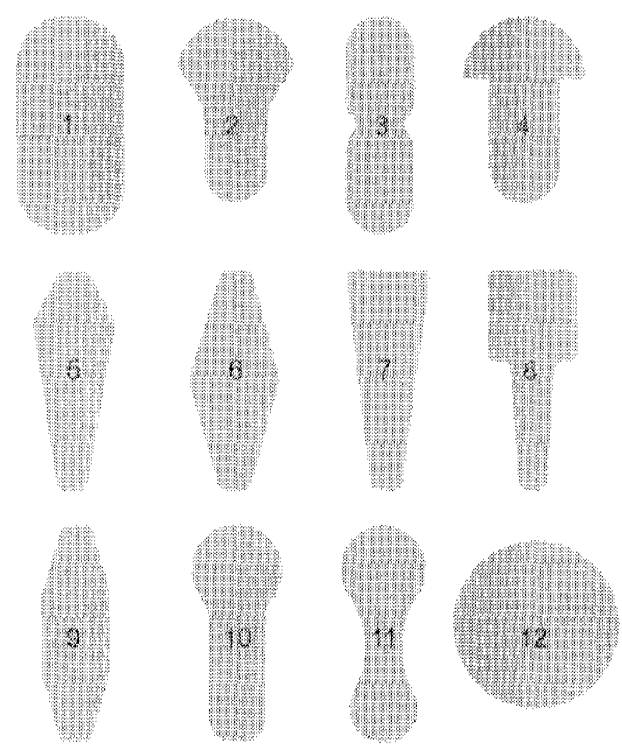

Figure 2: Stem geometry cross sections studied.

All models were simulated with the same loading configuration and same material properties. The comparisons of the predicted stresses for each of the problems simulated are a function of a unique design parameter (femoral stem cross-sectional geometry).

\section{Results and discussion}

As said elsewhere, the analysis was performed considering twelve different stem cross-sectional geometries with similar contact area. It is a controversy matter whether the critical region of stress in the prosthetic stem is within the mid-third of the stem or in the proximal region [1]. Our study is based on the premise that the proximal interfaces are the critical regions of the cemented hip replacement. The mean and the peak of the maximum (tension) and minimum (compression) principal stresses were obtained for the stem and at the bone-cement and cementstem interfaces, and will be simply referred as stresses (tension or compression). Since the stresses at the bone-cement interface are comparatively much lower 
than the ones at the cement-stem interface, only the last ones are shown and discussed.

Table 1. Area and second moment of area for the twelve sections studied.

\begin{tabular}{|c|c|c|c|c|}
\hline \multirow[b]{2}{*}{ Gevrnetry } & \multirow{2}{*}{$\begin{array}{c}\text { Area } \\
2 \\
\text { (min) }\end{array}$} & \multicolumn{3}{|c|}{ Second moment area ( $\left.\mathrm{rum}^{4}\right)$} \\
\hline & & $x x$ & yy & $z z$ \\
\hline 1 & $13636(-10)$ & 17073 & 80765 & 90751 \\
\hline 2 & $1380002)$ & 16035 & 73951 & 83765 \\
\hline 3 & $1355(-1.6)$ & 15045 & 61417 & 73744 \\
\hline 4 & $1354 \square(-1.7)$ & 11772 & 63058 & 70120 \\
\hline 5 & $13462(-20)$ & 13954 & 59666 & 70099 \\
\hline 6 & $13551(-1.6)$ & 14156 & 64357 & 75169 \\
\hline$?$ & $13770(00)$ & $1401 ?$ & 53193 & 6875 \\
\hline 8 & 138203 & 11505 & 53146 & 61787 \\
\hline$\underline{y}$ & $12270(-30)$ & 1355 & 58870 & 70077 \\
\hline 10 & $13715,-0.4$ & 15669 & 61733 & 74437 \\
\hline 11 & $13752(-0.1)$ & 15471 & 56678 & 69650 \\
\hline 12 & 13774 & 18399 & 98800 & 9880 \\
\hline
\end{tabular}

The stem cross-sectional geometry has a significant effect on the stresses within the stem and interfaces. Even thought the stresses at the stem are not relevant when compared to ones developed at the interfaces, we observed that the lowest stress value $(12 \mathrm{MPa})$ was induced by section $\# 2$, a typical Charnley crosssectional proximal geometry. On the other hand, section \#8 provoked the highest stress value, around $50 \mathrm{MPa}, 4$ times higher than the one obtained for section \#2.

A typical and common mode of femoral component loosening is related to the loss of proximal stem support [2] and has been associated with the failure of the bone-cement or cement-stem interfaces laterally due to excessive tensile stresses and fracture of the proximal cement medially due to excessive compression [1]. Figures 3 and 4 illustrate the mean and peak maximum principal stresses observed at the cement-stem interface for the lateral and posterior aspects of the implanted femur. Figures 5 and 6 illustrate the mean and peak minimum principal stresses at the same interface for the medial and anterior aspects.

Typically, the stresses developed at the bone-cement interface are much smaller then the ones developed at the cement-stem interface. In fact, for all aspects of the femur, and considering either tensile or compressive stresses, these were much higher at the cement-stem interface, which means that the bonding at this interface is more susceptible to failure. 


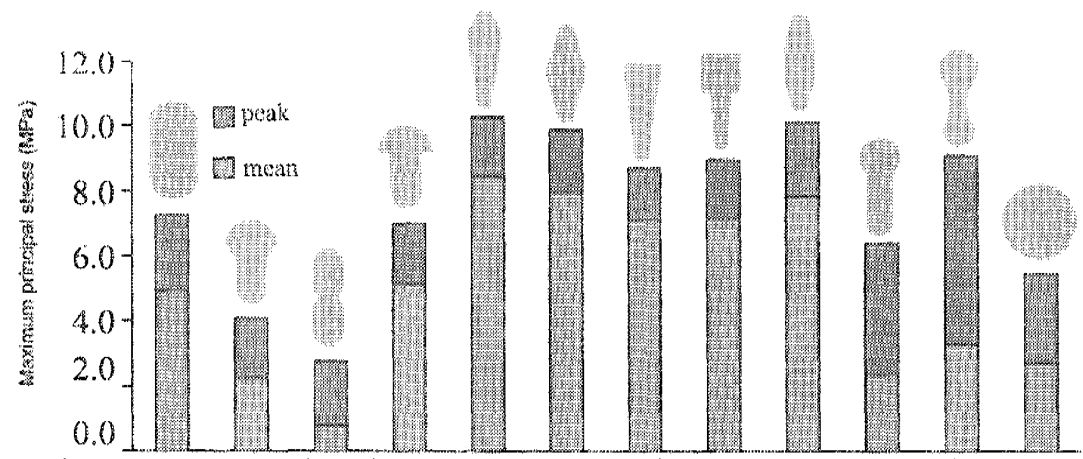

Figure 3: Mean and peak maximum principal stresses at the lateral aspect.

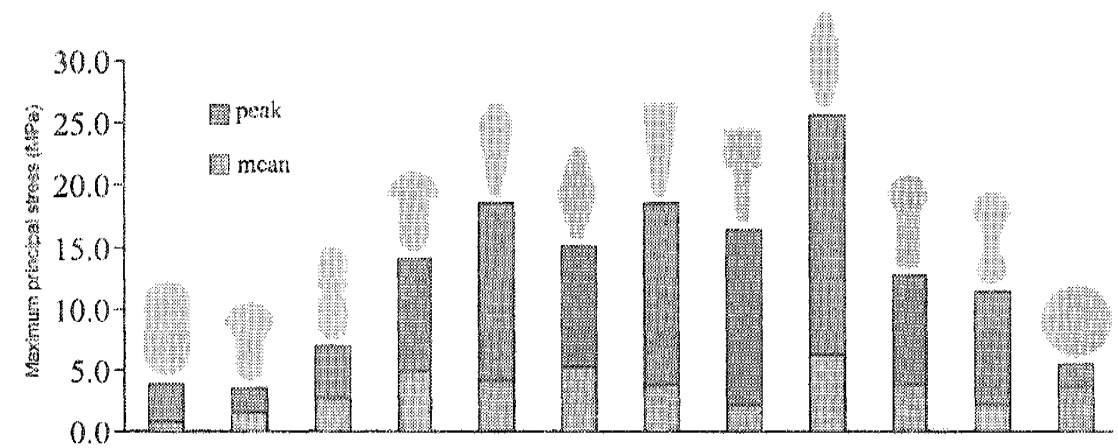

Figure 4: Mean and peak maximum principal stresses at the posterior aspect.

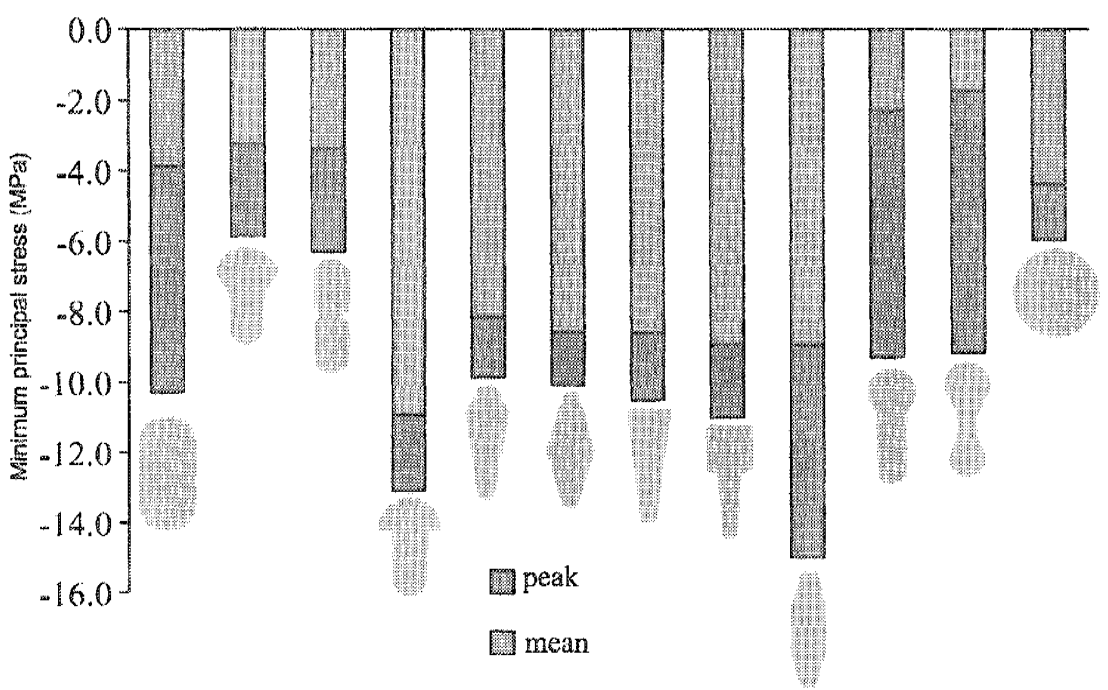

Figure 5: Mean and peak minimum principal stresses at the medial aspect. 


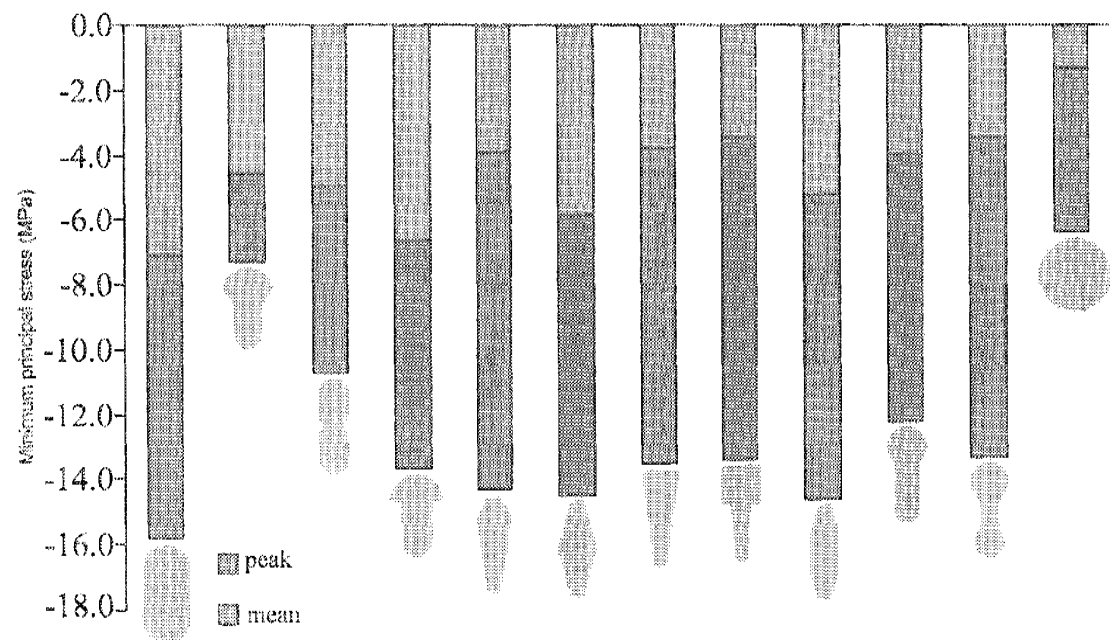

Figure 6: Mean and peak minimum principal stresses at the anterior aspect.

Overall, the mean and peak minimum compressive stresses are generally higher than the same for the maximum tensile stresses. At the lateral and posterior aspects of the femur, significant stress differences can be observed at the cementstem interface. At the lateral aspect, sharp rigid geometries (e.g. \#5, \#6, \#7, \#8 and \#9) provoke comparatively much higher mean and peak cement-stem stresses, of the order of 7-8 MPa and 9-10 MPa respectively. Organic geometries provoke stress values of the order of 1 to $3 \mathrm{MPa}$. For example, section \#2 (Charnley type) induces 4 times less stress values when compared with the sharp sections; section $\# 1$ and \#3 induce 8 times less stress levels. The peak stresses are slightly higher than the mean ones, around $2 \mathrm{MPa}$ for most of the geometries.

At the posterior aspect of the implanted femur, a similar behaviour was observed. However, the mean and peak stresses are higher. The stresses for sharp geometries are of the order $15 \mathrm{MPa}$. Section \#9 provoked the highest stress value $(25 \mathrm{MPa})$ stress value. Interesting to note that sections \#1, \#2 and \#3 provoked the lowest stresses for both the lateral and posterior aspects of the interfaces. Overall, section \#2 provoked a relatively low peak stress, 3-4 MPa. Sections \#1, $\# 2$, \#3, \#10 and \#11 apparently seam to be the best suited for the biomechanical problem simulated.

Similar conclusions can be observed at the medial and anterior aspects. The organic geometries induced much lower medial interface stresses, in a very similar way to the lateral interface. Again, sections \#1, \#2 and \#3 provoked lower stresses, of the order of $-4 \mathrm{MPa}$, while sections \#4, \#5, \#6, \#7, \#8 and \#9 provoked stresses of the order of $-10 \mathrm{MPa}$. Sections \#10 and \# 11 evidenced to be the best at the medial aspect of the implanted femur, since the stress levels are of the order of $-2 \mathrm{MPa}$. The peak stresses induced by sharp geometries are of the order of $10 \mathrm{MPa}$. The difference between the mean and peak stresses is small. At the anterior aspect of the cement-stem interface, we note more homogeneous 


\section{0}

Simulations in Biomedicine $V$

stress levels for all geometries, but no correlation can be drawn between the type of geometry and the stress levels. The stress values are of the order of -4 to -6 $\mathrm{MPa}$. A $14 \mathrm{MPa}$ peak stresses was observed for most of the cases simulated. Interesting to note that section \#2 induced the lowest stress levels at all interfaces.

Figure 7 illustrates the cross-sectional geometries in an increasing order of the mean maximum principal cement-stem interface stresses. Identical result was obtained for the minimum principal stresses.

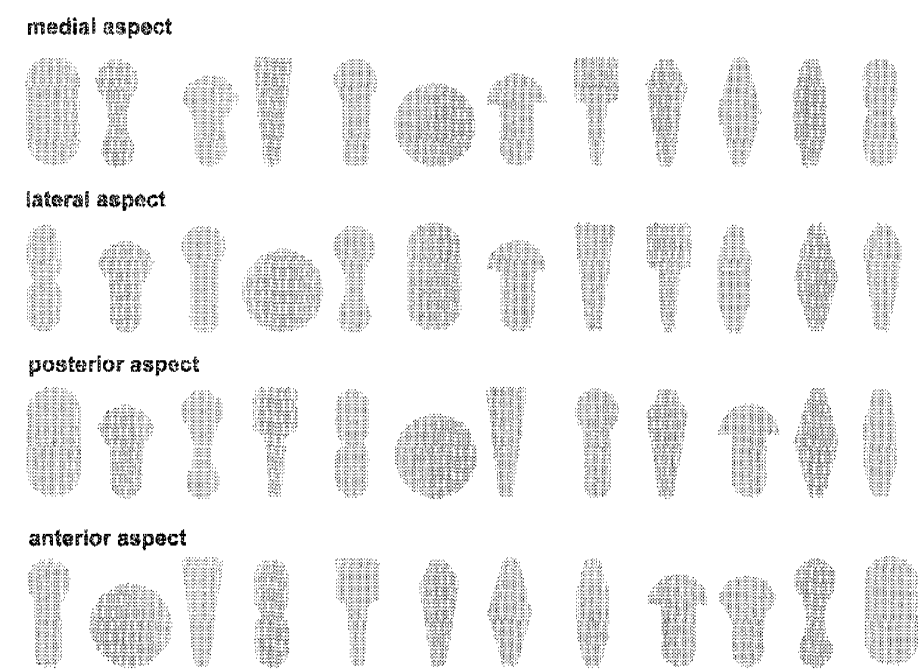

Figure 7: Cross-sectional geometries in an increasing order of the mean cement-stem interface stresses.

\section{Conclusions}

The studied herby described evidences the importance that the stem crosssectional geometry of the stem plays within the stresses developed at the cementstem interface. For the simplified model used, the results discussed evidence that the mean principal stresses are relevant at the lateral and medial aspects of the implanted femur; the peak stresses are important at the anterior and posterior aspects. However, these conclusions must be faced with necessary criticism, since they strongly depend on the numerical model used. Even though, they give us a tendency.

An important fact observed is that organic cross section geometries provoke much lower stresses. Other design factors, such as the medial curvature radius, can also play a decisive role in the implanted femur performance. A detailed study on the influence that these design parameters exert within the stem-bone load transfer mechanism will allow the development of new designs that can possibly perform better than many commercial cemented ones. The doubt is to 
know until what level is it actually possible to innovate within the design of a novel cemented hip femoral prosthesis?

\section{Acknowledgments}

The authors gratefully acknowledge the Fundação para a Ciência e a Tecnologia do Ministério da Ciência e do Ensino Superior for funding António Ramos through grant SFRH/BD/6317/2002 and project POCTI/EME/38367/2001.

\section{References}

[1] Crowninshield, R. D., Brand, R. A., Johnston, R. C. and Milroy, J. C., 1980, "The effect of femoral stem cross-sectional geometry on cement stresses in total hip reconstruction", Clin. Orthop. Rel. Res, 146, pp. 71.

[2] McNeice, G. J., and Gruen, T. A., 1976, "Mechanical failure modes of femoral components - radiographic examination of total hip replacement", Trans. 22 $2^{\text {nd }}$ Annual Orthop. Res. Soc., Vol. 1, pp. 1.

[3] Lee, A. J. C., Ling, R. S. M. and Wrighton, J. D., 1973, "Some properties of polymethylmethacrylate with reference to its use in orthopaedic surgery", clin. Orthop., 95, pp. 128.

[4] Freitag, T. A., and Cannon, S. L., 1977, "Fracture characterisitics of acrylic bone cements. II. Fatigue", J. Biomed. Mater. Res., 11, pp. 609.

[5] Fuch, H. O., 1965, "A set of fatigue failure criteria", Trans. ASME 87-D, pp. 333.

[5] Huiskes, R., Weinans, H., Grootenboer, H. J., Dalstra, M., fudala, B., Sloof, T. J., 1987, "adaptive bone remodelling theory applied to prosthetic-design analysis", J. Biomech., 20, pp. 1135.

[6] Kuiper, J. H., Huiskes, R., "Numerical optimization hip-prosthetic stem material", In Computer Methods in Biomechanics and Biomedicine (Eds. Middleton et al.), pp. 76, 1993 (Gordon and Breach, New York). 
